\title{
First principles materials design of novel functional oxides
}

\author{
Valentino R. Cooper ${ }^{\dagger} \|$, Brian K. Voas ${ }^{\dagger}$, Craig A. Bridges ${ }^{\S}$, \\ James R. Morris ${ }^{\dagger}$ and Scott P. Beckman ${ }^{\uparrow}$ \\ ${ }^{\dagger}$ Materials Science and Technology Division, Oak Ridge National Laboratory \\ Oak Ridge, TN 37831, USA \\ Department of Materials Science and Engineering \\ Iowa State University, Ames, IA 50011, USA \\ $\S_{\text {Chemical Sciences Division, Oak Ridge National Laboratory }}$ \\ Oak Ridge, TN 37831, USA \\ ISchool of Mechanical and Materials Engineering \\ Washington State University, Pulmann, WA 99196, USA \\ Icoopervr@ornl.gov
}

Received 30 January 2016; Revised 11 March 2016; Accepted 16 March 2016; Published 31 May 2016

\begin{abstract}
We review our efforts to develop and implement robust computational approaches for exploring phase stability to facilitate the prediction-to-synthesis process of novel functional oxides. These efforts focus on a synergy between (i) electronic structure calculations for properties predictions, (ii) phenomenological/empirical methods for examining phase stability as related to both phase segregation and temperature-dependent transitions and (iii) experimental validation through synthesis and characterization. We illustrate this philosophy by examining an inaugural study that seeks to discover novel functional oxides with high piezoelectric responses. Our results show progress towards developing a framework through which solid solutions can be studied to predict materials with enhanced properties that can be synthesized and remain active under device relevant conditions.
\end{abstract}

Keywords: First principles; materials design and discovery; oxide solid solutions.

\section{Introduction}

The past two decades have seen an unprecedented explosion in the use of computational methods in scientific research ranging from drug discovery to the design of new materials for electrodes in Li-ion batteries. This growth has largely been fueled by a steady increase in computational resources coupled with new algorithms that take advantage of larger, faster, massively parallelized computers. In the arena of materials research, these advances have made it possible to use first principles, electronic structure methods to explore the fundamental properties of realistic materials, at the level of atoms and electrons. ${ }^{1}$ Density functional theory (DFT) has had remarkable success for understanding how local interactions give rise to material responses, thus enabling the creation of design concepts for discovering new materials with enhanced behaviors. Moreover, once quantities relevant to macroscopic behaviors are known, high-throughput approaches that exploit the availability of massively parallel machines can be employed. ${ }^{2-10}$ In this way, a wealth of materials can be studied in a relatively short period of time. Data-mining strategies can then be used to extract the most promising materials based on data from crystal structure databases $^{11,12}$ and first principles calculations. Excellent examples of this strategy include the Materials Project, ${ }^{13}$ the
Combinatorial Sciences and Materials Informatics Collaboratory (CoSMIC), ${ }^{14}$ the Automated Interactive Infrastructure and Database for Computational Science (AiiDA) ${ }^{15}$ and the Automatic Flow for Materials Discovery (AFLOW), ${ }^{16}$ which have shown remarkable progress in the discovery of novel materials for battery cathodes, oxide sensors and scintillators, to name a few.

Here, we review our progress towards developing an approach for designing and discovering novel functional oxides for piezoelectric applications. The focus is on the use of first principles calculations to predict materials with enhanced properties that can be synthesized and remain active under device relevant conditions. The working postulate is that a comprehensive understanding of stability with respect to oxide impurity phases or end member compounds can facilitate the prediction-to-synthesis process. This is one facet of the Materials Genome Initiative which seeks to accelerate materials discovery. ${ }^{17}$ The standard model has been to (1) use computation to predict materials properties and then (2) to attempt to grow the most promising materials. The potential for discovering materials in this manner is great. However, materials predicted by theory/computation are often either difficult to synthesize, requiring extreme conditions such as high temperatures and pressures, or are simply unstable and

This is an Open Access article published by World Scientific Publishing Company. It is distributed under the terms of the Creative Commons Attribution 4.0 (CC-BY) License. Further distribution of this work is permitted, provided the original work is properly cited. 


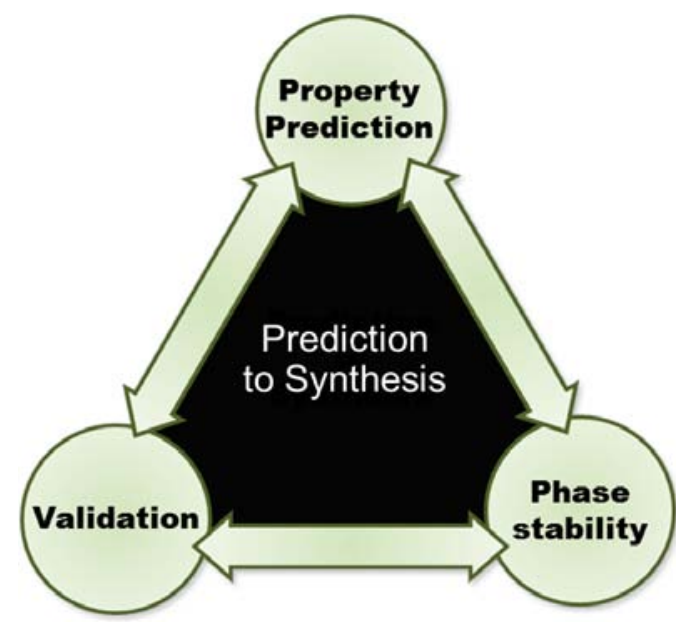

Fig. 1. Prediction-to-synthesis triangle illustrating the three fundamental pillars of the computational materials discovery process: (i) Prediction of materials with desired properties, (ii) assessment of phase stability, both with regards to structural transitions and competing phases and (iii) experimental validation. This approach focuses on the computational examination of the relative stability of competing phases or impurities in order to shine a light on the feasibility of synthesizing a compound as well as its operability under specific conditions, which is often overlooked in the materials prediction process. Yet, routine assessment is necessary for discovering new materials.

thus segregate into secondary phases. Experimental feedback can be used to train the computational sets, but this is time consuming and can be quite computationally expensive. A third pillar of the prediction-to-synthesis triangle (see Fig. 1) should therefore include a theoretical assessment of the phase stability of predicted materials. Identification of the energetics of competing phases with different structures and stoichiometries will not only give a clear understanding of which structure is the ground state, but may give insight into which routes need to be taken to prepare the desired material. This has the potential for reducing the steps needed to get from properties prediction to experimental realization. Unfortunately, an understanding of the complex energy landscape of many materials remains elusive and is one of the great challenges in theoretical condensed matter physics today. ${ }^{18}$

In this paper, we present an example study which integrates properties prediction, experimental validation and phase stability to develop and build upon chemical concepts to design new materials with enhanced properties in the context of piezoelectrics. In particular, we will examine our efforts to map out the phase diagram of a disordered, perovskite oxide solid solution to identify regions of high piezoresponse that may be amenable to experimental synthesis. We will discuss the use and development of techniques to treat chemical disorder, relative stability of competing phases and ferroelectric phase transitions. Ultimately, we intend to present a framework through which solid solutions of oxides can be routinely studied to give experimentally realizable predictions.

\section{Designer Solid Solutions}

A useful strategy for creating high piezoresponse compounds borrows lessons from the prototypical piezoelectric $\mathrm{Pb}(\mathrm{Zr}, \mathrm{Ti})$ $\mathrm{O}_{3}$ (PZT), a solid solution of the tetragonally distorted $\mathrm{PbTiO}_{3}(\mathrm{PT})$ and the anti-ferrodistorted $\mathrm{PbZrO}_{3}(\mathrm{PZ})$. The key to the success of PZT lies in the combination of perovskite compounds with two very distinct phases; leading to a composition-dependent region, the morphotropic phase boundary (MPB) at a concentration of roughly 50\% PT/50\% PZ. Here, the crystal structure undergoes a transition (mediated by a monoclinic phase ${ }^{19}$ ) between the macroscopic phases of the parent compounds ${ }^{20}$ (i.e., the rhombohedral phase of the PZ-rich compositions and the tetragonal phase of the PT-rich compositions). In general, ferroelectric solid solutions near MPBs exhibit enhanced electromechanical responses when the parent compounds have high remnant polarizations. ${ }^{21}$ In this respect, the discovery of new MPB systems is an important step towards engineering high, electromechanical responses. The driving force of these high responses is often a result of competition between local strain or local bonding frustration and long range polar behavior. ${ }^{22}$

As such, theoretical methods capable of probing local structure interactions are crucial for a precise understanding of the link between local structure and macroscopic properties. For example, using first principles studies we demonstrated that it is the cooperative off-centering of the $A$ - and $B$-site ions that give rise to the MPB and large piezoelectric response of PZT. ${ }^{23,24}$ In particular, $\mathrm{Pb}$ displacements were observed to be extremely sensitive to the local $B$-site ordering, displacing away from the larger $\mathrm{Zr}$ cations and towards the smaller $\mathrm{Ti}$ ions, while conforming to the overall polarization. Hence, the relative concentration of $\mathrm{Ti}$ versus $\mathrm{Zr}$ critically defines the direction of the polarization; rotating from tetragonal to rhombohedral with increasing amounts of $\mathrm{Zr}$ cations. The challenge in developing new materials is finding combinations of $A$ - and $B$-site cations that allow us to manipulate short-range behavior while maintaining the ferroelectric perovskite structure.

\subsection{Properties prediction}

In an attempt to design a novel lead-free piezoelectric based on the above chemical concept, we initially explored solid solutions of $\mathrm{Bi}\left(\mathrm{Zn}_{1 / 2} \mathrm{Ti}_{1 / 2}\right) \mathrm{O}_{3}$ (BZT) and $\mathrm{La}\left(\mathrm{Zn}_{1 / 2} \mathrm{Ti}_{1 / 2}\right) \mathrm{O}_{3}$ (LZT). Similar to PT, BZT has a super-tetragonal ground state, ${ }^{25}$ while LZT is anti-ferroelectric ${ }^{26}$ like PZ. Table 1 lists lattice constants and polarizations for the end member BZT and LZT bulk oxides. All calculations employed $2 \times 2 \times 2$ unit cells with rock salt ordering of $\mathrm{Zn}$ and $\mathrm{Ti}$ on the $B$-site. A tendency towards this ordering was recently demonstrated in BZT. ${ }^{27}$ It should be noted that the DFT supercell calculations are the main bottleneck for obtaining the results discussed in this paper. A typical perovskite supercell self-consistent (scf) static calculation requred approximately 7 minutes on 
128 cores of a Cray XT system (i.e., Hopper at NERSC). A full relaxation of atomic coordinates and lattice constants may require a few 10 s of scf steps. In principal, on a large cluster, jobs with different cation arrangements and compositions can be performed simultaneously. Nevertheless, a high-throughput study of the system described above may take on the order of a week to complete.

To search for a MPB in this compound we examined the polarization as a function of $\mathrm{Bi}$ versus $\mathrm{La}$ fraction assuming, to the best that it could have been maintained, rock salt ordering on the $A$-site (Fig. 2). This was done to reduce any artificial symmetry breaking due to $A$-site ordering. Subsequent calculations that mimic disorder revealed very little dependence on the $A$-site ordering. Here, we find that, for large fractions of $\mathrm{La}$, the polarization remains very low, with marginal rhombohedral distortions. On the other hand for large fractions of $\mathrm{Bi}$ we observe a huge increase in the polarization with large tetragonal-like distortions and the absence of a monoclinic phase. In both cases, these results present unfavorable conditions for a high performance, room temperature ferroelectric. On the La-rich part of the phase diagram, the low polarizations may translate into extremely low transition temperatures as suggested by recent work by Grinberg and collaborators that used Landau theory to demonstrate that the magnitude of the polarization is correlated with the ferroelectric transition temperature. ${ }^{28,29}$ Conversely, the high polarizations in the Bi-rich region of the phase

Table 1. Lattice constant $\left(a_{\mathrm{o}}\right), c / a$ ratio and polarization for the end members of the ternary BZT-LZT-PT solid solution. Experimental values ${ }^{25,26}$ are listed in parentheses.

\begin{tabular}{lccc}
\hline System & $a_{\mathrm{o}}[\AA]$ & $c / a$ & $P\left[\mathrm{C} / \mathrm{m}^{2}\right]$ \\
\hline LZT & $3.91(3.95)$ & $1.00(1.00)$ & $0.00(0.00)$ \\
BZT & $3.74(3.82)$ & $1.23(1.211)$ & $1.42(1.50)$ \\
PT & 3.85 & 1.05 & 0.79 \\
\hline
\end{tabular}

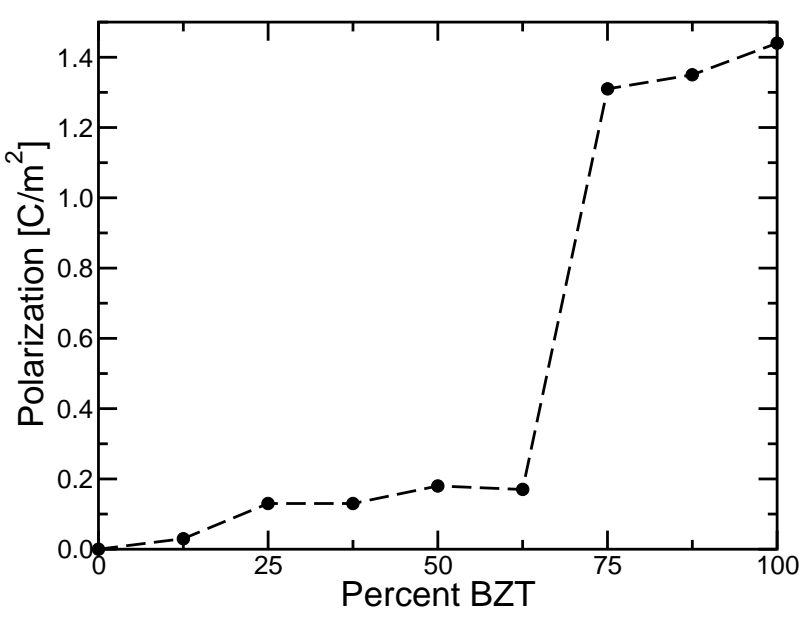

Fig. 2. BZT-LZT solid solution polarization as a function of $\% \mathrm{Bi}^{34}$ diagram suggest that these compositions may not be ferroelectric as such large polarizations are often associated with loss in switchability and piezoelectric response as seen in compressively strained, epitaxial $\mathrm{BiFeO}_{3} \cdot{ }^{30-33}$

To reduce the polarization of the $\mathrm{Bi}$-rich region, and to improve solubility, ${ }^{35}$ we examined the ternary phase diagram for solid solutions with PT. It is known that throughout the BZT-PT phase diagram the material remains tetragonal, with PT decreasing the magnitude of polarization. We found that in the BZT-LZT-PT ternary phase diagram, La plays two specific roles: (1) rotating the polarization away from the tetragonal direction towards the rhombohedral direction (for moderate amounts of LZT) and (2) reducing the magnitude of the polarization. Figure 3 depicts the magnitude and rotation of the polarization away from the tetragonal direction for the BZT-LZT-PT ternary solid solution. Here, we see a clear reduction in the total polarization with $<75 \%$ LZT compositions having reasonable polarizations. Furthermore, we note the presence of a monoclinic phase for PT concentrations between $25 \%$ and $50 \%$.

\subsection{Modeling solid solutions}

Predicting the properties of oxide solid solutions requires the ability to find the ground state properties of a wide range of multi-atomic systems. The predicted ground state can be significantly influenced by the arrangement and compositional fraction of cations as well as the choice of unit cell or crystal structure. DFT supercell size constraints present additional limitations, particularly with regards to the treatment of disorder. To address the challenge of modeling disordered solid solutions, several approaches have been pursued to explore the rich landscape of structure- and compositiondependent properties. Atomic averaging approaches where individual atoms are viewed as either a combination of two or more atoms (virtual crystal approximation; $\mathrm{VCA}^{36,37}$ ) or only seeing an average local environment (like the coherent potential approximation; $\mathrm{CPA}^{38,39}$ ) have been successful in describing the properties of a number of systems.

In oxide ferroelectrics, such as the aforementioned PZT, these mean-field techniques often fail to capture the shortrange local interactions that drive macroscopic phenomena such as polarization rotation and electromechanical responses. ${ }^{24,40,41}$ First principles methods can, however, be employed to parameterize larger unit cells or phenomenological models to study the effects of local variations in cation arrangements and composition. To date, these methods have given significant insight into the physical properties of a number of perovskite systems and when combined with molecular dynamics (MD) or Monte Carlo (MC) simulations can be used to explore temperature-dependent phase transitions and domain wall motion. ${ }^{42-47}$ Unfortunately, the extensive effort required to parameterize these potentials from large experimental or computational datasets has limited their widespread application. 


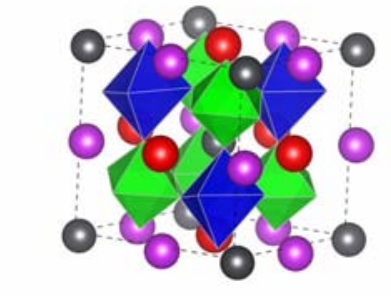

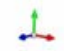

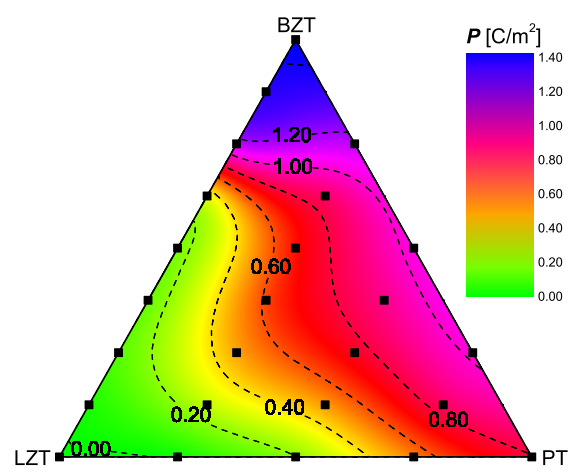

(a)

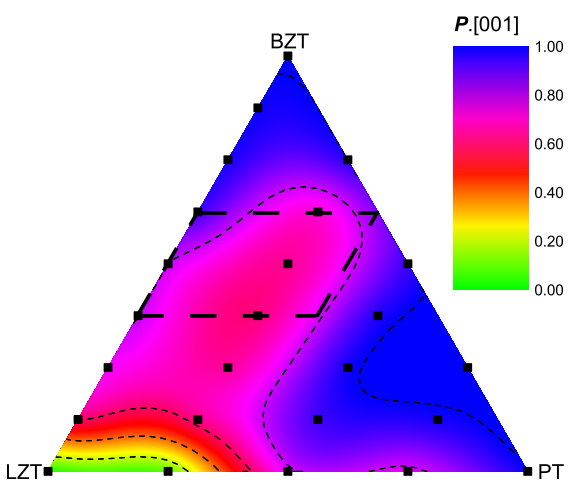

(b)

Fig. 3. (Color online) (Left) Atomic arrangement for a $3 \mathrm{Bi}(\mathrm{Zn}, \mathrm{Ti}) \mathrm{O}_{3}-3 \mathrm{La}(\mathrm{Zn}, \mathrm{Ti}) \mathrm{O}_{3}-2 \mathrm{PbTiO}_{3}$ supercell. Green and blue polyhedra denote $\mathrm{Ti}$ and $\mathrm{Zn}$ octahedra, respectively. Pink, red and black atoms represent $\mathrm{Bi}, \mathrm{La}$ and $\mathrm{Pb}$, respectively. (Right) BZT-LZT-PT ternary diagrams for (a) $|P|$ and (b) P.[001]. For the P.[001] ternary diagram, the blue, purple and pink regions indicate areas that are tetragonal, monoclinic and rhombohedral, respectively. The green regions have little or no polarization. Black squares denote the compositions modeled. The dashed parallelogram represents the region of high stability. Adapted from Ref. 24. Copyright (2012) American Chemical Society.

An alternative is the special quasirandom structure $(\mathrm{SQS})^{48}$ method in which a supercell is selectively populated such that the short-ranged, geometric correlations approximate that of the random alloy. This approach has been widely used in the metals ${ }^{49-51}$ and semiconductors ${ }^{52,53}$ communities. Recently, using the SQS approach we examined the high temperature $(R 3 c)$ and low temperature $(\mathrm{Pm})$ phases of the $\mathrm{KNbO}_{3}-\mathrm{NaNbO}_{3}(\mathrm{KNO}-\mathrm{NNO})$ solid solution. ${ }^{54}$ Similar to PZT, KNO-NNO shows a significant enhancement in piezoelectric response at a composition near $\left(\mathrm{K}_{0.5} \mathrm{Na}_{0.5}\right) \mathrm{NbO}_{3}$ $(\mathrm{KNN})^{55}$ and therefore has been considered to be an excellent starting structure for designing high-response, lead-free, electroceramics. ${ }^{56-58}$ Given the relative chemical similarities between $\mathrm{K}$ and $\mathrm{Na}$, it is anticipated that these cations will be distributed randomly on the perovskite $A$-site. Figure 4 depicts the SQS and rock salt ordered structure (RSS) used to simulate KNN. In short, we find that the pair distribution functions (PDFs) generated with the SQS method were in excellent agreement with experimental PDFs for the two phases. This was in stark contrast with the RSS, which is often used to study these materials. ${ }^{59}$ Analyses of the SQS polarization direction indicated that the macroscopic phase depends on the local, nearest-neighbor, $\mathrm{Na}-\mathrm{O}$ interactions. These bond changes exhibit the most significant differences between the ground state $R 3 \mathrm{c}$ and room temperature $\mathrm{Pm}$ phases; thus implicating $\mathrm{Na}$ interactions as the driving force for the observed electromechanical responses.

Theory and experiment have demonstrated that in materials with large size and charge differences on the $B$-site sub-lattice there may be a tendency towards $B$-cation ordering. This is often seen in $\mathrm{Nb}$ containing systems such as the relaxor systems $\mathrm{Pb}(\mathrm{Zn}, \mathrm{Nb}) \mathrm{O}_{3}, \mathrm{~Pb}(\mathrm{Mg}, \mathrm{Nb}) \mathrm{O}_{3}$, and $\mathrm{Pb}(\mathrm{Sc}, \mathrm{Nb})$ $\mathrm{O}_{3} \cdot{ }^{60-65}$ Such behavior was also observed in the $\mathrm{Bi}(\mathrm{Zn}, \mathrm{Nb})$ $\mathrm{O}_{3}$-based systems. ${ }^{27}$ For the BZT-LZT-SrTiO 3 (ST) solid solutions that we studied, ${ }^{66}$ a comparison between the SQS and RSS arrangements of $\mathrm{Zn}$ and $\mathrm{Ti}$ revealed that in the majority of instances the $B$-site RSS was the lowest energy structure. On the other, hand we found very little dependence of the total energy on the arrangement of cations on the $A$-site. Hence, for these systems and the BZT-LZT-PT systems we explored only the RSS arrangements.

Approaches like the SQS method and exploiting tendencies towards ordering are invaluable tools in the search and discovery of novel oxide solid solutions. Together, they facilitate the simulation of solid solutions, which would ordinarily require a large number of atoms, in manageable supercells; thereby allowing for realistic screening of materials and material properties.

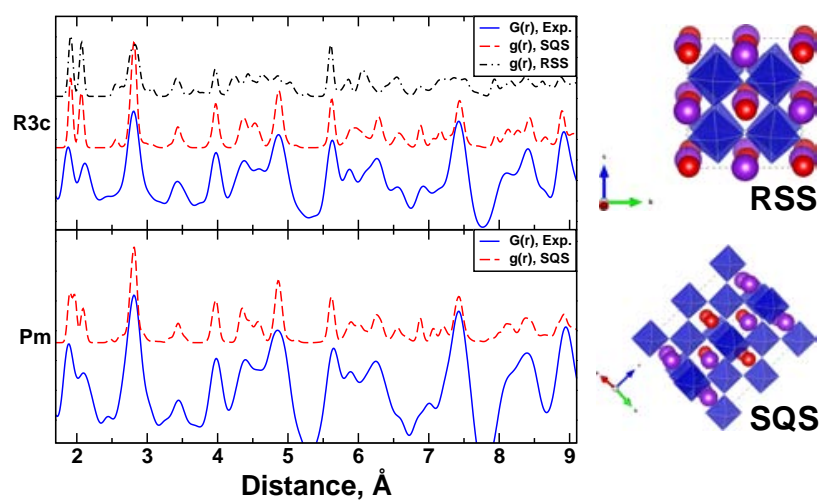

Fig. 4. (Color online) (Left) The experimental (blue solid line) and theoretical reduced pair distribution functions for the high temperature $R 3 c$ (top) and low temperature $P m$ (bottom) phases of KNN. Rock salt (RSS) ordered and special quasirandom (SQS) structures are indicated by red dashed lines and black dash-dot lines, respectively. (Right) RSS and SQS atomic structures used to study the $R 3 c$ phase of KNN. Printed with permission from Ref. 54. Copyright (2014) American Physical Society. 


\subsection{Phase stability and experimental validation}

Tremendous progress has been made in the field of modeling the thermodynamic stability of metal alloys. ${ }^{67-69}$ The combination of first principles calculations and thermodynamic/ statistical methods with mean-field methods and the aforementioned SQS technique have allowed computational scientists to discern which metal alloys will form and thus may be suitable for synthesis. ${ }^{70-76}$ A pertinent example is the most recent work that investigated the electronic and structural properties of high entropy alloys. ${ }^{77}$ Using enthalpies of formation computed directly from DFT and an ideal entropy of mixing model, they were able to build a model to identify all known single-phase alloys relative to those known not to form. Furthermore, they were able to extend this methodology to predict new high entropy alloys that had not been previously investigated.

To interrogate phase stability versus phase segregation into end member compounds, the Gibbs free energy, $\Delta G$, upon mixing must be evaluated as:

$$
\Delta G=\Delta H-T \Delta S .
$$

$\Delta H$, the enthalpy of mixing, can be computed directly from first principles calculations of the difference in total energy between the final solid solution or alloy and the end member compounds. $T$ is temperature and $\Delta S$ is the change in entropy. In the case of these oxides configurational effects dominate, allowing us to use the entropy of mixing associated with an ideal random mixture, which can be evaluated as:

$$
\Delta S=-k_{\mathrm{B}} \sum_{i} x_{i} \ln x_{i},
$$

where $x_{i}$ is the fraction of the $i$ th end member compound in the solid solution or alloy and $k_{\mathrm{B}}$ is the Boltzmann constant.

We applied this approach to explore the phase stability of oxide solid solutions of BZT-LZT-PT ${ }^{34}$ and BZT-LZTST. ${ }^{66}$ Figure 5 depicts the Gibbs free energy evaluated with the inclusion of configurational entropy as a function of BZT content for a binary solid solution of BZT-PT for different values of $k_{\mathrm{B}} \mathrm{T}$. To better understand the feasibility of synthesis, we compute the temperature (in meV) at which $\Delta G=0$ (Fig. 5 right). (This provides a simple approach to identifying which compositions are likely to form; in reality, equilibrium is determined by the choice of phase or phases that minimize the Gibbs free energy.) For the BZT-PT phase diagram, the $k_{\mathrm{B}} T$ required to stabilize the solid solution is relatively small near the end members and increases to $\sim 50 \mathrm{meV}$ for a $50 / 50$ composition. This phase stability range correlates well with a previous report on the $x \mathrm{Bi}\left(\mathrm{Mg}_{1 / 2} \mathrm{Ti}_{1 / 2}\right) \mathrm{O}_{3}-y \mathrm{Bi}\left(\mathrm{Zn}_{1 / 2} \mathrm{Ti}_{1 / 2}\right)$ $\mathrm{O}_{3}-z \mathrm{PbTiO}_{3}$ perovskite ternary solid solution where mixed phases (i.e., phase segregation) were observed for BZT-PT compositions with greater than $40 \% \mathrm{BZT}^{78}$ and with other attempts to grow pure phases of BZT-PT. ${ }^{79,80}$

Conversely, for the BZT-LZT solid solution (Fig. 5, right) we find that $\mathrm{Bi}$ is mostly insoluble in LZT and La also phase segregates in BZT, again, in agreement with experimental observations. ${ }^{35}$ Our calculations further predict that for BZTLZT, compositions between $37.5 \%$ and $62.5 \%$ LZT should be suitable for forming solid solutions. To test this theory, we attempted to make a 50\% BZT/50\% LZT solid solution. A stoichiometric mixture of $\mathrm{Bi}_{2} \mathrm{O}_{3}$ (Aldrich, 99.999\%), $\mathrm{La}_{2} \mathrm{O}_{3}$ (Alfa Aesar, 99.99\%), $\mathrm{TiO}_{2}$ (Alfa Aesar, 99.9\%) and $\mathrm{ZnO}$ (Alfa Aesar, 99.99\%) were ground and pelletized using a cold press. The sample was fired at $750^{\circ} \mathrm{C}(5 \mathrm{~h}), 800^{\circ} \mathrm{C}(10 \mathrm{~h})$, $850^{\circ} \mathrm{C}(10 \mathrm{~h})$, and $900^{\circ} \mathrm{C}(10 \mathrm{~h})$ in an alumina crucible with intermediate regrinding and pelletizing. Powder X-ray diffraction data were obtained with a PANalytical Empyrian diffractometer using $\mathrm{Cu} \mathrm{K} \alpha$ radiation, and were collected in Bragg-Brentano geometry on a flat plate holder. Figure 6 shows the X-ray crystal diffraction, XRD, spectra for a trial synthesis of BZT-LZT. The diffraction pattern shows peaks consistent with a cubic or pseudo-cubic perovskite structure. The pattern does not appear consistent with
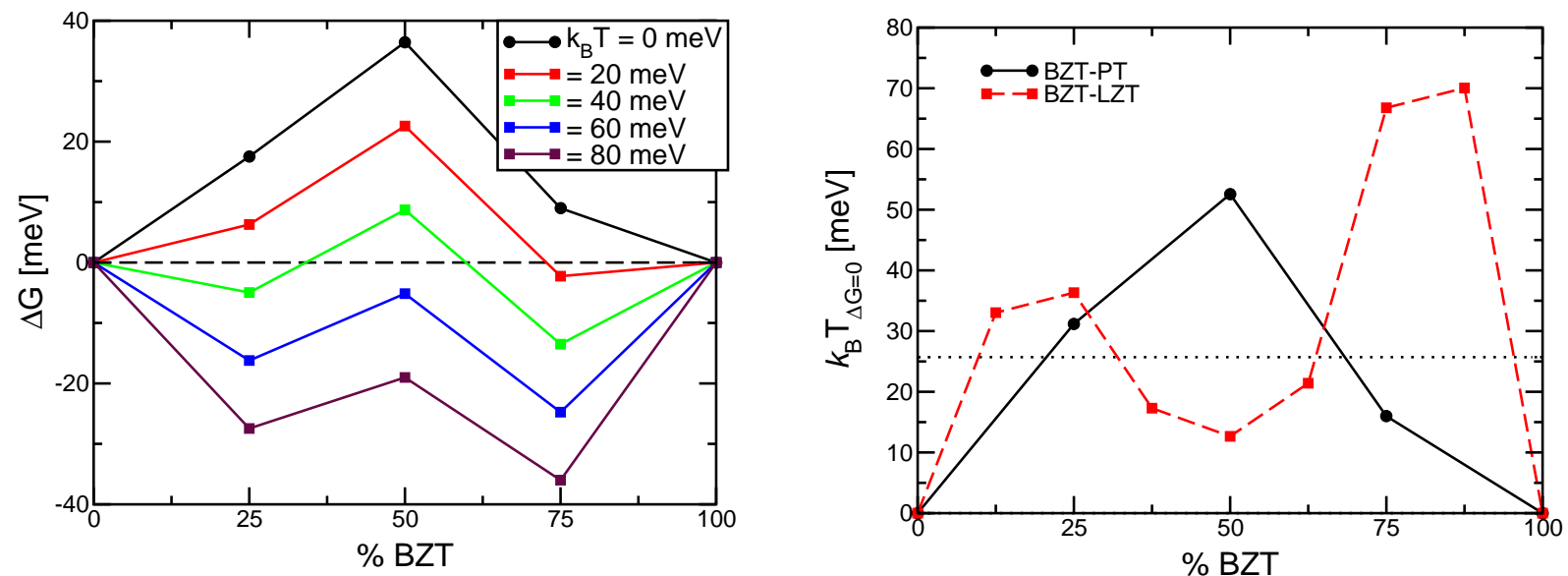

Fig. 5. (Left) Gibbs free energy, $\Delta G$ for different temperatures (in units of $k_{\mathrm{B}} T$ [meV]) as a function of $\%$ BZT for the BZT-PT phase diagram. (right) Temperature (in units of $k_{\mathrm{B}} T[\mathrm{meV}]$ ) at which $\Delta G=0$ for the BZT-PT and BZT-LZT phase diagrams. The dotted line indicates $k_{\mathrm{B}} T$ at room temperature, below which it is assumed that a solid solution will form. 
potential non-bismuth containing perovskite phases such as $\mathrm{La}_{2 / 3} \mathrm{TiO}_{3}$ (ICSD 236451), $\mathrm{LaTiO}_{3}$ (ICSD 63575) or reported monoclinic $\mathrm{La}_{2} \mathrm{ZnTiO}_{6}$ (ICSD 172755). Although some impurities, most likely excess Bi oxides, are present, these initial synthesis efforts signal promise for growing solid solutions of this phase. A more conclusive structural assignment will require further work to identify a phase pure region of the phase diagram.

An analysis of $\Delta G$ of the ternary BZT-LZT-PT solid solutions reveals two regions of stability. The first is for compositions with high PT content. Again, this is in good agreement with experimental results. ${ }^{79,80}$ The second region is a band between $37.5 \%$ and $67.5 \%$ BZT and $<67.5 \%$ PT concentrations (see Fig. 3(b)). This band overlays the monoclinic region as seen in Fig. 3(b) and may be a suitable region to explore for experimental synthesis efforts.

In their entirety, our initial theoretical and experimental attempts demonstrate that, similar to work on metal alloy systems, the configurational entropy is essential for identifying stable solid solutions which may be missed with a simple enthalpy of mixing calculation. Of course, other factors may contribute to phase stability. For example, the theoretical results presented here are relative to the end members of BZT and PT and exclude the possibility of $\mathrm{PbO}_{2}$ (Scrutinyte) and $\mathrm{Bi}$ oxide compounds (e.g., Sillenite or Aurivillius forms), which are commonly observed in experiment. ${ }^{78}$ Additionally, preparation conditions may play a role. Pure BZT requires high pressure synthesis (up to $6 \mathrm{GPa}$ ); thus, it stands to reason that solid solutions in the BZT-LZTPT or BZT-LZT-ST phase diagrams with high BZT content may require similar synthesis conditions to be realized.

A final point to consider is temperature- (or pressure-) dependent phase transitions. Ferroelectric perovskites at high temperatures present a cubic, nonpolar phase. The lack of polarization in this phase renders them unusable as piezoelectrics at this temperature and thus the onset of this transition is critical to device performance. To elucidate the ferroelectric transition temperature, $T_{c}$, MC or MD simulations can be used to explore phase transition temperatures and the onset of ferroelectricity. However, such calculations,

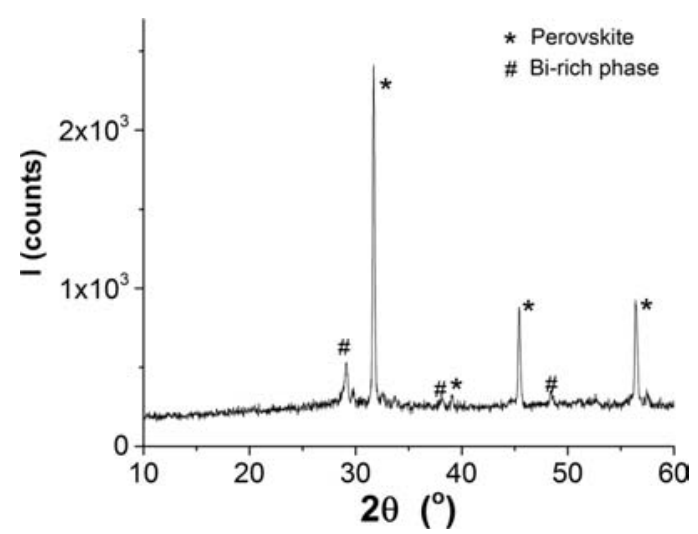

Fig. 6. XRD spectra for the $50 \% \mathrm{BZT} / 50 \% \mathrm{LZT}$ solid solution.
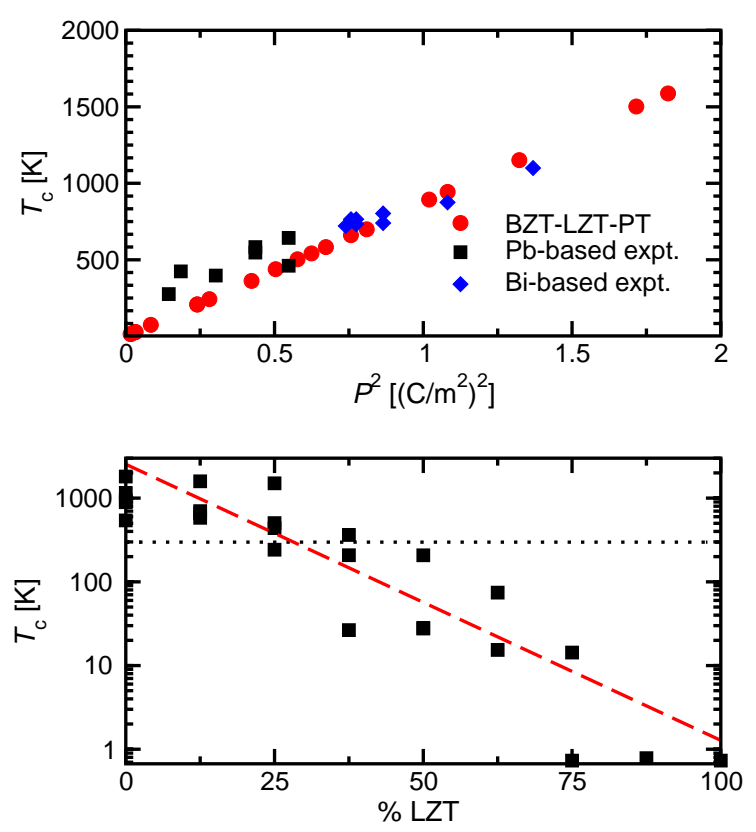

Fig. 7. (Color online) (Top) $T_{c}$ versus $P^{2}$ for the BZT-LZT-PT solid solution (red circles). For comparison we plot the predicted polarizations versus the experimental $T_{c} s$ for a range of $\mathrm{Pb}$-based ${ }^{28}$ and Bi-based ${ }^{29}$ compounds. (bottom) Predicted $T_{c} s$ as a function of LZT concentration for the BZT-LZT-PT solid solution. The dotted line indicates room temperature, while the red dashed line is a fit through the data emphasizing the correlation between $T_{c}$ and LZT content.

directly from first principles results, are time consuming and require large unit cells or parameterized potentials. Using Landau theory, a clear correlation between experimental ferroelectric $T_{c} s$ and computed polarizations, $T_{c}=\gamma P^{2}$, can be obtained. ${ }^{28,29}$ Using this relationship (with $\gamma=870 \mathrm{Km}^{4} / \mathrm{C}^{2}$ ) we approximated $T_{c} s$ for our Bi-based compounds. Fig. 7 (top) depicts the predicted $T_{c}$ versus the DFT computed polarization. For comparison, we plot the DFT computed polarization versus the experimental $T_{c} s$ previously obtained for a set of $\mathrm{Pb}$-based ${ }^{28}$ and Bi-based ${ }^{29}$ ferroelectric compounds. Remarkable agreement between the newly predicted results and the previous data can be seen. Interestingly, we also observe a strong dependence on La content (see Fig. 7, bottom). This is perhaps easily understood from Fig. 1 where we see that it is only after a considerable decrease in La content that the BZT-LZT solid solution recovers polarizations similar to that of the BZT end member. This indicates that La not only results in a rotation of polarization from [001] in these materials, but also restricts the total polarization by limiting the overall off-center displacements of the cations within the material; thereby being the driving force for tuning the ferroelectric transition temperature.

On the basis of our examination of the magnitude and orientation of the polarization as well as our predicted $T_{c}$ and phase stability, we speculate that the region of the phase diagram bounded by $25 \% \leq \mathrm{PT} \leq 50 \%$ and $50 \% \leq \mathrm{LZT} \leq$ $25 \%$ should be the most likely compositions to find a high 
response piezoelectric (see Fig. 3). In this region, we find suitably high polarizations $\left(0.71 \mathrm{C} / \mathrm{m}^{2}<\mathrm{P}<1.14 \mathrm{C} / \mathrm{m}^{2}\right)$ with moderate phase stability and appropriate $\mathrm{T}_{c}>500 \mathrm{~K}$. Recent experimental efforts to grow regions of this ternary phase diagram further emphasize the importance of dialog between theory and experiment. In this regard, the evaluation of phase stability plays a crucial role in facilitating these interactions.

\section{Conclusions and Outlook}

The desire to accelerate materials discovery has propelled materials research in many new and fascinating directions. The need to understand the complex landscape of multicomponent/multiphase materials not only presents new challenges for computational materials design, but it also provides new opportunities for the development of novel computational techniques. Data mining and high-throughput strategies have emphasized the value of electronic structure approaches for quickly predicting new materials. Methods like DFT have excelled at making accurate predictions of $0 \mathrm{~K}$ ground state properties, while finite temperature properties may be explored using empirical potentials and MD and/or MC simulations. Similarly, thermodynamic models, like those that provide approximations for the entropy of mixing as an ideal random mixture, allow for the use of electronic structure energies to assess whether a material can be feasibly synthesized. These methods have been widely applied to metal alloy systems and, when coupled with techniques that can efficiently model disorder (e.g., the SQS approach) or search configurational space (e.g., genetic algorithms, simulated annealing, etc.), they provide a basis for discerning ground state materials properties. These approaches are now being applied to oxides and are yielding promising results. For instance, using SQS it is possible to gain insights into the driving mechanisms behind the transition between the lowtemperature and high-temperature phases of the $\left(\mathrm{K}_{1 / 2} \mathrm{Na}_{1 / 2}\right)$ $\mathrm{NbO}_{3}$ solid solution. ${ }^{54}$ Additionally, predictions of phase stability point to potentially synthesizable regions of the $\mathrm{Bi}$ $(\mathrm{Zn}, \mathrm{Ti}) \mathrm{O}_{3}-\mathrm{La}(\mathrm{Zn}, \mathrm{Ti}) \mathrm{O}_{3}-\mathrm{PbTiO}_{3}$ phase diagram ${ }^{34}$ (some of which have already been confirmed by experiment).

Ferroelectric oxides, such as the above-mentioned solid solutions, have a wide range of properties that involve coupling between electric, magnetic and lattice degrees of freedom. These behaviors are often influenced by environmental conditions such as temperature and pressure. While estimates of the temperature at which a material transforms from a high-temperature cubic, paraelectric phase to a ferroelectric, polar phase can be inferred based on the polarization of the ground state phase, further challenges remain. For instance many of these materials undergo a series of phase transitions as they cool down to 0K. Mapping out the pressure- and temperature-dependent phase diagram presents a unique and complex challenge, particularly for solid solutions where it may not be trivial to parameterize empirical potentials or effective Hamiltonians. For now, these endeavors may be limited due to the computational demands. However, as computer processing power continues to increase and more efficient algorithms are developed, it may become possible in the near future to perform MC or MD simulations where the energy is evaluated directly from first principles calculations in reasonably sized supercells, thereby opening the door to mapping out the complex energy landscape of materials directly from electronic structure calculations.

More importantly the synergy between theory and experiment provides an opportunity for discovering new materials faster than in previous decades. Routine evaluations of phase stability and transition temperatures along with ground state properties signal an era of computational materials design of realistic, synthesizable materials that will remain active under device relevant conditions. This will reduce the cost needed to iterate through the prediction-tosynthesis cycle aiding in an accelerated materials discovery process, one that may take months rather than years.

\section{Acknowledgments}

B.K.V. acknowledges summer support through the HERE program at ORNL. Research supported by the US Department of Energy (DOE), Office of Science, Basic Energy Sciences (BES), Materials Sciences and Engineering Division (VRC, JRM), and the Office of Science Early Career Research Program (VRC). SPB acknowledges support from the US National Science Foundation under Grant No. DMR-1037898. CAB acknowledges support from the Laboratory Directed Research and Development program of Oak Ridge National Laboratory, managed by UT-Battelle, LLC, for the U.S. Department of Energy. This research used resources of the National Energy Research Scientific Computing Center, which is supported by the Office of Science of the US Department of Energy under Contract No. DE-AC02-05CH11231.

\section{References}

${ }^{1}$ DOE, Directing matter and energy: Five challenges for science and the imagination (2007).

${ }^{2}$ R. Armiento, B. Kozinsky, M. Fornari and G. Ceder, Screening for high-performance piezoelectrics using high-throughput density functional theory, Phys. Rev. B 84, 014103 (2011).

${ }^{3}$ S. R. Broderick, H. Aourag and K. Rajan, Classification of oxide compounds through data-mining density of states spectra, J. Am. Ceram. Soc. 94, 2974 (2011).

${ }^{4}$ S. R. Broderick, H. Aourag and K. Rajan, Data mining density of states spectra for crystal structure classification: An inverse problem approach, Stat. Anal. Data Min. 1, 353 (2009).

${ }^{5}$ G. Hautier, C. C. Fischer, A. Jain, T. Mueller and G. Ceder, Finding natures missing ternary oxide compounds using machine learning and density functional theory, Chem. Mater. 22, 3762 (2010). 
${ }^{6}$ A. Jain, G. Hautier, C. J. Moore, S. P. Ong, C. C. Fischer, T. Mueller, K. A. Persson and G. Ceder, A high-throughput infrastructure for density functional theory calculations, Comput. Mater. Sci. 50, 2295 (2011).

${ }^{7}$ B. Kang and G. Ceder, Battery materials for ultrafast charging and discharging, Nature 458, 190 (2009).

${ }^{8}$ D. Morgan, A. Van der Ven and G. Ceder, Li conductivity in $\mathrm{Li}_{x} M \mathrm{PO}_{4}(M=\mathrm{Mn}, \mathrm{Fe}, \mathrm{Co}, \mathrm{N})$ olivine materials, Electrochem. Solid-State Lett. 7, A30 (2004).

${ }^{9}$ S. P. Ong, L. Wang, B. Kang and G. Ceder, Li-Fe-P-O 2 phase diagram from first principles calculations, Chem. Mater. 20, 1798 (2008).

${ }^{10}$ H. Zenasni, H. Aourag, S. R. Broderick and K. Rajan, Electronic structure prediction via data-mining the empirical pseudopotential method, Phys. Status Solidi B 247, 115 (2010).

${ }^{11}$ Cambridge Structural Database (CSD), http://www.ccdc.cam.ac.uk/.

${ }^{12}$ Inorganic Crystal Structure Database (ICSD), https://icsd.fizkarlsruhe.de/.

${ }^{13}$ A. Jain, S. P. Ong, G. Hautier, W. Chen, W. D. Richards, S. Dacek, S. Cholia, D. Gunter, D. Skinner, G. Ceder and K. A. Persson, The materials project: A materials genome approach to accelerating materials innovation, APL Materials 1(1), 011002 (2013).

${ }^{14}$ Combinatorial Sciences and Materials Informatics Collaboratory (CoSMIC), http://cosmic.mse.iastate.edu/.

${ }^{15}$ G. Pizzi, A. Cepellotti, R. Sabatini, N. Marzari and B. Kozinsky, AiiDA: Automated interactive infrastructure and database for computational science, Comp. Mat. Sci. 111, 218 (2016).

${ }^{16}$ Automatic Flow for materials discovery, (Aflow) http://www. aflowlib.org/.

${ }^{17}$ Materials Genome Initiative (MGI), https://www.whitehouse.gov/ mgi.

${ }^{18}$ S. M. Woodley and R. Catlow, Crystal structure prediction from first principles, Nat. Mater. 7, 937 (2008).

${ }^{19}$ B. Noheda, D. E. Cox, G. Shirane, J. A. Gonzalo, L. E. Cross and S.-E. Park, A monoclinic ferroelectric phase in the $\mathrm{Pb}\left(\mathrm{Zr}_{1-x} \mathrm{Ti}_{x}\right)$ $\mathrm{O}_{3}$ solid solution, Appl. Phys. Lett. 74, 2059 (1999).

${ }^{20} \mathrm{H}$. Fu and R. E. Cohen, Polarization rotation mechanism for ultrahigh electromechanical response in single-crystal piezoelectrics, Nature 403, 281 (2000).

${ }^{21}$ R. Guo, L. E. Cross, S.-E. Park, B. Noheda, D. E. Cox and G. Shirane, Origin of the high piezoelectric response in $\mathrm{PbZr}_{1-x} \mathrm{Ti}_{x} \mathrm{O}_{3}$, Phys. Rev. Lett. 84, 5423 (2000).

${ }^{22}$ M. Ghita, M. Fornari, D. J. Singh and S. V. Halilov, Interplay between $A$-site and $B$-site driven instabilities in perovskites, Phys. Rev. B 72, 054114 (2005).

${ }^{23}$ V. R. Cooper, I. Grinberg, N. R. Martin and A. M. Rappe, Local structure of PZT, AIP Conf. Proc., Vol. 626(2002), p. 26.

${ }^{24}$ I. Grinberg, V. R. Cooper and A. M. Rappe, Relationship between local structure and phase transitions of a disordered solid solution, Nature 419, 909 (2002).

${ }^{25}$ M. R. Suchomel, A. M. Fogg, M. Allix, H. Niu, J. B. Claridge and M. J. Rosseinsky, $\mathrm{Bi}_{2} \mathrm{ZnTiO}_{6}$ : A lead-free closed-shell polar perovskite with a calculated ionic polarization of $150 \mu \mathrm{C} / \mathrm{cm}^{-2}$, Chem. Mater. 18, 4987 (2006).

${ }^{26}$ A. Aguadero, J. Alonso, M. Martínez-Lope and M. Fernández-Díaz, Crystallo-chemical evolution of the $\mathrm{La}_{2} \mathrm{ZnTiO}_{6}$ double perovskite upon reduction: A structural study, Solid State Sci. 13, 13 (2011).

${ }^{27}$ T. Qi, I. Grinberg and A. M. Rappe, First-principles investigation of the highly tetragonal ferroelectric material $\mathrm{Bi}\left(\mathrm{Zn}_{1 / 2} \mathrm{Ti}_{1 / 2}\right) \mathrm{O}_{3}$, Phys. Rev. B 79, 094114 (2009).
${ }^{28}$ I. Grinberg and A. M. Rappe, Local structure and macroscopic properties in $\mathrm{PbMg}_{1 / 3} \mathrm{Nb}_{2 / 3} \mathrm{O}_{3}-\mathrm{PbTiO}_{3}$ and $\mathrm{PbZn}_{1 / 3} \mathrm{~N}_{2 / 3} \mathrm{O}_{3}$ $\mathrm{PbTiO}_{3}$ solid solutions, Phys. Rev. B 70, 220101(R) (2004).

${ }^{29}$ I. Grinberg and A. M. Rappe, Nonmonotonic $T_{c}$ trends in Bi-based ferroelectric perovskite solid solutions, Phys. Rev. Lett. 98, 037603 (2007).

${ }^{30}$ R. J. Zeches, M. D. Rossell, J. X. Zhang, A. J. Hatt, Q. He, C.-H. Yang, A. Kumar, C. H. Wang, A. Melville, C. Adamo, G. Sheng, Y.-H. Chu, J. F. Ihlefeld, R. Erni, C. Ederer, V. Gopalan, L. Q. Chen, D. G. Schlom, N. A. Spaldin, L. W. Martin and R. Ramesh, A strain-driven morphotropic phase boundary in $\mathrm{BiFeO}_{3}$, Science 326, 977 (2009).

${ }^{31}$ C. Beekman, W. Siemons, T. Z. Ward, J. D. Budai, J. Z. Tischler, R. Xu, W. Liu, N. Balke, J. H. Nam and H. M. Christen, Unit cell orientation of tetragonal-like $\mathrm{BiFeO}_{3}$ thin films grown on highly miscut $\mathrm{LaAlO}_{3}$ substrates, Appl. Phys. Lett. 102, 221910 (2013).

${ }^{32}$ Z. Chen, Z. Luo, C. Huang, Y. Qi, P. Yang, L. You, C. Hu, T. Wu, J. Wang, C. Gao, T. Sritharan and L. Chen, Low-symmetry monoclinic phases and polarization rotation path mediated by epitaxial strain in multiferroic $\mathrm{BiFeO}_{3}$ thin films, Adv. Funct. Mater. 21, 133 (2011).

${ }^{33}$ H. Béa, B. Dupé, S. Fusil, R. Mattana, E. Jacquet, B. WarotFonrose, F. Wilhelm, A. Rogalev, S. Petit, V. Cros, A. Anane, F. Petroff, K. Bouzehouane, G. Geneste, B. Dkhil, S. Lisenkov, I. Ponomareva, L. Bellaiche, M. Bibes and A. Barthélémy, Evidence for room-temperature multiferroicity in a compound with a giant axial ratio, Phys. Rev. Lett. 102, 217603 (2009).

${ }^{34}$ V. R. Cooper, J. R. Morris, S. Takagi and D. J. Singh, La-driven morphotropic phase boundary in the $\mathrm{Bi}\left(\mathrm{Zn}_{1 / 2} \mathrm{Ti}_{1 / 2}\right) \mathrm{O}_{3}$ $\mathrm{La}\left(\mathrm{Zn}_{1 / 2} \quad \mathrm{Ti}_{1 / 2}\right) \mathrm{O}_{3}-\mathrm{PbTiO}_{3}$ solid solution, Chem. Mater. 24, 447 (2012).

${ }^{35}$ Private communication with Z.-G. Ye.

${ }^{36}$ N. J. Ramer and A. M. Rappe, Virtual-crystal approximation that works: Locating a compositional phase boundary in $\mathrm{Pb}(\mathrm{Zr}, \mathrm{Ti}) \mathrm{O}_{3}$, Phys. Rev. B Rapid Commun. 62, R743 (2000).

${ }^{37}$ L. Bellaiche and D. Vanderbilt, Virtual crystal approximation revisited: Application to dielectric and piezoelectric properties of perovskites, Phys. Rev. B 61, 7877 (2000).

${ }^{38}$ G. M. Stocks and H. Winter, Self-consistent-field-Korringa-KohnRostoker-coherent-potential approximation for random alloys, Z. Phys. B 46, 95 (1982).

${ }^{39}$ G. M. Stocks and A. Gonis (eds.), Alloy Phase Stability (Kluwer Academic, Dordrecht, 1989).

${ }^{40}$ R. E. Cohen, Origin of ferroelectricity in perovskite oxides, Nature 358, 136 (1992).

${ }^{41}$ C. G. F. Stenger and A. J. Burggraaf, Order-disorder reactions in the ferroelectric perovskites $\mathrm{Pb}\left(\mathrm{Sc}_{1 / 2} \mathrm{Nb}_{1 / 2}\right) \mathrm{O}_{3}$ and $\mathrm{Pb}\left(\mathrm{Sc}_{1 / 2} \mathrm{Ta}_{1 / 2}\right)$ $\mathrm{O}_{3}$. II. Relation between ordering and properties, Phys. Status Solidi A 61, 33 (1980).

${ }^{42}$ S. Tinte, M. G. Stachiotti, M. Sepliarsky, R. L. Migoni and C. O. Rodriquez, Atomistic modeling of $\mathrm{BaTiO}_{3}$ based on first-principles calculations, J. Phys.: Condens. Matter 11, 9679 (1999).

${ }^{43}$ M. Sepliarsky, S. R. Phillpot, M. G. Stachiotti and R. L. Migoni, Ferroelectric phase transitions and dynamical behavior in $\mathrm{KNbO}_{3}$ $/ \mathrm{KTaO}_{3}$ superlattices by molecular-dynamics simulation, J. Appl. Phys. 91, 3165 (2002).

${ }^{44}$ S. Tinte, J. Íñiguez, K. M. Rabe and D. Vanderbilt, Quantitative analysis of the first-principles effective Hamiltonian approach to ferroelectric perovskites, Phys. Rev. B 67, 064106 (2003). 
${ }^{45}$ I. Grinberg, V. R. Cooper and A. M. Rappe, Oxide chemistry and local structure of $\mathrm{PbZr}_{x} \mathrm{Ti}_{1-x} \mathrm{O}_{3}$ studied by density-functional theory supercell calculations, Phys. Rev. B 69, 144118 (2004).

${ }^{46}$ L. Walizer, S. Lisenkov and L. Bellaiche, Finite-temperature properties of $(\mathrm{Ba}, \mathrm{Sr}) \mathrm{TiO}_{3}$ systems from atomistic simulations, Phys. Rev. B 73, 144105 (2006).

${ }^{47}$ Y.-H. Shin, I. Grinberg, I.-W. Chen and A. M. Rappe, Nucleation and growth mechanism of ferroelectric domain-wall motion, $\mathrm{Na}$ ture 449, 06165 (2007).

${ }^{48}$ A. Zunger, S. H. Wei, L. G. Ferreira and J. E. Bernard, Special quasirandom structures, Phys. Rev. Lett. 65, 353 (1990).

${ }^{49}$ A. van de Walle, G. Ceder and U. V. Waghmare, First-principles computation of the vibrational entropy of ordered and disordered $\mathrm{Ni}_{3} \mathrm{Al}$, Phys. Rev. Lett. 80, 4911 (1998).

${ }^{50}$ J. von Pezold, A. Dick, M. Friák and J. Neugebauer, Generation and performance of special quasirandom structures for studying the elastic properties of random alloys: Application to Al-Ti, Phys. Rev. B 81, 094203 (2010).

${ }^{51}$ J. Frantti, Y. Fujioka and R. M. Nieminen, Pressure-induced phase transitions in $\mathrm{PbTiO}_{3}$ : A query for the polarization rotation theory, J. Phys. Chem. B 111, 4287 (2007).

${ }^{52}$ A. M. Saitta, S. de Gironcoli and S. Baroni, Structural and electronic properties of a wide-gap quaternary solid solution: (Zn, Mg) (S, Se), Phys. Rev. Lett. 80, 4939 (1998).

${ }^{53}$ S.-H. Wei and A. Zunger, Giant and composition-dependent optical bowing coefficient in GaAsN alloys, Phys. Rev. Lett. 76, 664 (1996).

${ }^{54}$ B. K. Voas, T.-M. Usher, X. Liu, S. Li, J. L. Jones, X. Tan, V. R. Cooper and S. P. Beckman, Special quasirandom structures to study the $\left(\mathrm{K}_{0.5} \mathrm{Na}_{0.5}\right) \mathrm{NbO}_{3}$ random alloy, Phys. Rev. B 90, 024105 (2014).

${ }^{55}$ Y.-J. Dai, X.-W. Zhang and K.-P. Chen, Morphotropic phase boundary and electrical properties of $\mathrm{K}_{1-x} \mathrm{Na}_{x} \mathrm{NbO}_{3}$ lead-free ceramics, Appl. Phys. Lett. 94, 042905 (2009).

${ }^{56}$ C. W. Ahn, S. Y. Lee, H. J. Lee, A. Ullah, J. S. Bae, E. D. Jeong, J. S. Choi, B. H. Park and I. W. Kim, The effect of K and Na excess on the ferroelectric and piezoelectric properties of $\mathrm{K}_{0.5} \mathrm{Na}_{0.5} \mathrm{NbO}_{3}$ thin films, J. Phys. D 42, 215304 (2009).

${ }^{57}$ J. Tellier, B. Malic, B. Dkhil, D. Jenko, J. Cilensek and M. Kosec, Crystal structure and phase transitions of sodium potassium niobate perovskites, Solid State Sci. 11, 320 (2009).

${ }^{58}$ L. Wu, J. L. Zhang, C. L. Wang and J. C. Li, Influence of compositional ratio $\mathrm{K} / \mathrm{Na}$ on physical properties in $\left(\mathrm{K}_{x} \mathrm{Na}_{1-x}\right) \mathrm{NbO}_{3}$ ceramics, J. Appl. Phys. 103, 084116 (2008).

${ }^{59}$ S. P. Beckman, X. Wang, K. M. Rabe and D. Vanderbilt, Ideal barriers to polarization reversal and domain-wall motion in strained ferroelectric thin films, Phys. Rev. B 79, 144124 (2009).

${ }^{60}$ H. B. Krause and D. L. Gibbon, Z. Kristallogr. 134, 44 (1971).

${ }^{61}$ A. D. Hilton, D. J. Barber, C. A. Randall and T. R. Shrout, On short range ordering in the perovskite lead magnesium niobate, $J$. Mater. Sci. 25, 3461.

${ }^{62}$ P. K. Davies and M. A. Akbas, Chemical order in PMN-related relaxors: Structure, modification, and impact on properties, J. Phys. Chem. Solids 61, 159 (2000).

${ }^{63}$ S. A. Prosandeev, E. Cockayne, B. P. Burton, S. Kamba, J. Petzelt, Y. Yuzyuk, R. S. Katiyar and S. B. Vakhrushev, Lattice dynamics in $\mathrm{PbMg}_{1 / 3} \mathrm{Nb}_{2 / 3} \mathrm{O}_{3}$, Phys. Rev. B 70, 134110 (2004).

${ }^{64}$ F. Chu, I. M. Reaney and N. Setter, Spontaneous (zero-field) relaxor-to-ferroelectric-phase transition in disordered $\mathrm{Pb}$ $\left(\mathrm{Sc}_{1 / 2} \mathrm{Nb}_{1 / 2}\right) \mathrm{O}_{3}$, J. Appl. Phys. 77, 1671 (1995).
${ }^{65}$ C. Malibert, B. Dkhil, J. M. Kiat, D. Durand, J. F. Bérar and A. Spasojevic-de Biré, Order and disorder in the relaxor ferroelectric perovskite $\mathrm{Pb}\left(\mathrm{Sc}_{1 / 2} \mathrm{Nb}_{1 / 2}\right) \mathrm{O}_{3}$ ( $\mathrm{PSN}$ ): Comparison with simple perovskites $\mathrm{BaTiO}_{3}$ and $\mathrm{PbTiO}_{3}$, J. Phys.: Condens. Matter 9, 7485 (1997)

${ }^{66}$ B. K. Voas, S. P. Beckman and V. R. Cooper, Morphotropic phase boundary in a lead free ferreoelectric solid solution: $\mathrm{Bi}\left(\mathrm{Zn}_{1 / 2} \mathrm{Ti}_{1 / 2}\right)$ $\mathrm{O}_{3}-\mathrm{La}\left(\mathrm{Zn}_{1 / 2} \mathrm{Ti}_{1 / 2}\right) \mathrm{O}_{3}-\mathrm{SrTiO}_{3}$, unpublished (2016).

${ }^{67}$ A. van de Walle and M. Asta, First-Principles Modeling of Phase Equilibria, Handbook of Materials Modeling, ed. S. Yip (Springer, 2005), p. 349.

${ }^{68}$ M. Asta, V. Ozolins and C. Woodward, A first-principles approach to modeling alloy phase equilibria, JOM 53, 16 (2001).

${ }^{69}$ M. Asta, M. van Schilfgaarde and D. de Fontaine, A first-principles study of the phase stability of fcc-and hcp-based Ti-Al alloys, MRS Proc., eds. I. Baker, J. Whittenberger, R. Darolia and M. Yoo, MRS Online Proceedings Library Archive, Vol. 288 (1992), p. 153.

${ }^{70} \mathrm{G}$. Rubin and A. Finel, Application of first-principles methods to binary and ternary alloy phase diagram predictions, J. Phys.: Condens. Matter 7, 3139 (1995).

${ }^{71}$ D. Andersson, P. Korzhavyi and B. Johansson, First-principles based calculation of binary and multicomponent phase diagrams for titanium carbonitride, Calphad 32, 543 (2008).

${ }^{72}$ W. P. Huhn, M. Widom and M. C. Gao, First principles modeling of the temperature dependent ternary phase diagram for the $\mathrm{Cu}$ Pd-S system, Comput. Mater. Sci. 92, 377 (2014).

${ }^{73}$ A. V. Ruban and V. I. Razumovskiy, First-principles based thermodynamic model of phase equilibria in bcc Fe-Cr alloys, Phys. Rev. B 86, 174111 (2012).

${ }^{74}$ D. Reith, M. Stöhr, R. Podloucky, T. C. Kerscher and S. Müller, First-principles modeling of temperature- and concentration-dependent solubility in the phase-separating alloy $\mathrm{Fe}_{x} \mathrm{Cu}_{1-x}$, Phys. Rev. B 86, 020201 (2012).

${ }^{75}$ S. Zhou, Y. Wang, C. Jiang, J. Zhu, L.-Q. Chen and Z.-K. Liu, First-principles calculations and thermodynamic modeling of the Ni-Mo system, Mater. Sci. Eng. A 397, 288 (2005).

${ }^{76}$ V. Ozolinšs, C. Wolverton and A. Zunger, $\mathrm{Cu}-\mathrm{Au}, \mathrm{Ag}-\mathrm{Au}, \mathrm{Cu}-\mathrm{Ag}$, and $\mathrm{Ni}-\mathrm{Au}$ intermetallics: First-principles study of temperaturecomposition phase diagrams and structures, Phys. Rev. B 57, 6427 (1998).

${ }^{77}$ M. C. Troparevsky, J. R. Morris, P. R. C. Kent, A. R. Lupini and G. M. Stocks, Criteria for predicting the formation of single-phase high-entropy alloys, Phys. Rev. X, 011041 (2015).

${ }^{78}$ A. Dwivedi, W. Qu and C. A. Randall, Preparation and characterization of high-temperature ferroelectric $x \mathrm{Bi}\left(\mathrm{Mg}_{1 / 2} \mathrm{Ti}_{1 / 2}\right) \mathrm{O}_{3^{-}}$ $y \mathrm{Bi}\left(\mathrm{Zn}_{1 / 2} \mathrm{Ti}_{1 / 2}\right) \mathrm{O}_{3}-z \mathrm{PbTiO}_{3}$ perovskite ternary solid solution, J. Am. Ceram. Soc. 94, 4371 (2011).

${ }^{79}$ M. R. Suchomel and P. K. Davies, Enhanced tetragonality in $(x)$ $\mathrm{PbTiO}_{3}-(1-x) \mathrm{Bi}\left(\mathrm{Zn}_{1 / 2} \mathrm{Ti}_{1 / 2}\right) \mathrm{O}_{3}$ and related solid solution systems, Appl. Phys. Lett. 86, 262905 (2005).

${ }^{80}$ Z. Yao, K. Lu, C. Xu, H. Hao, Q. Xu, Z. Song, Z. Yu, A. Ullah and $\mathrm{H}$. Liu, La-driven variation in structure and property of $(\mathrm{Bi}, \mathrm{La})$ $\left(\mathrm{Zn}_{0.5} \mathrm{Ti}_{0.5}\right) \mathrm{O}_{3}-\mathrm{PbTiO}_{3}$ high-temperature piezoceramics, J. Alloys Compd. 663, 41 (2016). 